\title{
Disconnected contributions to D-meson semi-leptonic decay form factors
}

\author{
G. S. Bali ${ }^{a}$, S. Collins ${ }^{a}$, R. Horsley ${ }^{b}$, I. Kanamori ${ }^{* a}$, Y. Nakamura ${ }^{c}$, D. Pleiter ${ }^{a, d}$, \\ P. Pérez-Rubio ${ }^{a}$, P. E. L. Rakow ${ }^{e}$, A. Schäfer ${ }^{a}$, G. Schierholz ${ }^{f}$, F. Winter ${ }^{b}$, \\ J. M. Zanotti ${ }^{g}$ (QCDSF Collaboration) \\ ${ }^{a}$ Institut für Theoretische Physik, Universität Regensburg, D-93040 Regensburg, Germany. \\ ${ }^{b}$ School of Physics, University of Edinburgh, Edinburgh EH9 3JZ, UK. \\ ${ }^{c}$ RIKEN Advanced Institute for Computational Science, Kobe, Hyogo 650-0047, Japan. \\ ${ }^{d}$ Jülich Supercomputing Centre, Forschungszentrum Jülich, D-52425 Jülich, Germany. \\ ${ }^{e}$ Theoretical Physics Division, Department of Mathematical Sciences, University of Liverpool, \\ Liverpool L69 3BX, UK. \\ ${ }^{f}$ Deutsches Elektronen-Synchrotron DESY, 22603 Hamburg, Germany. \\ ${ }^{g}$ Special Research Centre for the Subatomic Structure of Matter, School of Chemistry \& Physics, \\ University of Adelaide, South Australia 5005, Australia. \\ E-mail: issaku.kanamori@physik.uni-regensburg.de
}

\begin{abstract}
We calculate the disconnected contribution to the form factor for the semileptonic decay of a $D$-meson into a final state, containing a flavor singlet eta meson. We use QCDSF $n_{f}=2+1$ configurations at the flavor symmetric point $m_{u}=m_{d}=m_{s}$ and the partially quenched approximation for the relativistic charm quark. Several acceleration and noise reduction techniques for the stochastic estimation of the disconnected loop are tested.
\end{abstract}

The XXIX International Symposium on Lattice Field Theory - Lattice 2011

July 10-16, 2011

Squaw Valley, Lake Tahoe, California

*Speaker. 


\section{Introduction}

Semileptonic decays of $D$-mesons contain rich physics. Lattice calculations of the form factors for these decays are important for the search for hints of new physics through the determination of CKM matrix elements. These form factors have been well-studied on the lattice. Previously, some of us tested a stochastic method to measure 3-point functions needed to calculate the semileptonic decay form factor [1]. The advantage of stochastic methods is that we have access to a greater range of momenta at fixed cost. This enables us to extract the form factor more reliably from results for the three point functions at different momentum transfers.

In particular, the $D_{s}$ meson is interesting for flavor physics. Its major semi-leptonic decay is to $\eta$ and $\eta^{\prime}$, which has a contribution from a disconnected loop diagram (Fig. 1). The loop runs over three light flavors so the effect is enhanced by a factor three, and thus may be large. The purpose of this work is to test the feasibility of measuring the disconnected diagram, and to quantify its contribution to the form factor.

We extract the scalar form factor $f_{0}$ from the relation [2]:

$$
f_{0}\left(q^{2}\right)=\frac{m_{c}-m_{l}}{m_{D_{s}}^{2}-m_{\eta}^{2}}\left\langle\eta|S| D_{s}\right\rangle
$$

where $S=\bar{l} c$ is a scalar current made from charm and light quarks. $m_{i}$ are the masses of the quarks and mesons. The matrix element can be extracted from the following ratio of 3-point over 2-point functions:

$$
\left\langle\eta\left(\vec{k}, t_{i}\right)|S(\vec{q}, t)| D_{s}\left(\vec{p}, t_{f}\right)\right\rangle=Z_{\eta} Z_{D_{s}} \frac{C_{3}\left(t_{f}-t_{i}, t-t_{i} ; \vec{p}, \vec{q}\right)}{C_{2}^{\eta}\left(t-t_{i} ; \vec{k}\right) C_{2}^{D_{s}}\left(t_{f}-t ; \vec{p}\right)}=Z_{\eta} Z_{D_{s}} R\left(\vec{k}, \vec{q}, \vec{p}, t-t_{i}, t_{f}-t_{i}\right),
$$

and similarly for $\eta^{\prime}$. For large $t_{f}-t_{i}$ and $t-t_{i}$ this ratio should approach a constant. $Z_{\eta}$ and $Z_{D_{s}}$ are the overlap factors between the meson state and the interpolating operator, which can be extracted from the two point functions $C_{2}^{\eta}\left(t-t_{i} ; \vec{k}\right)$ and $C_{2}^{D_{s}}\left(t_{f}-t ; \vec{p}\right)$, respectively. The two point functions for $\eta$ and $\eta^{\prime}$ also have a disconnected part, however, at $m_{\mathrm{PS}} \simeq 445 \mathrm{MeV}$ we expect its contribution to the mass to be small and we neglect it in this first exploratory study.

We use QCDSF $24^{3} \times 48 n_{f}=2+1$ configurations [3]. So far we only use the SU(3) symmetric set ( $\kappa_{l}=\kappa_{s}=0.1209$ ) with lattice spacing $a \simeq 0.08 \mathrm{fm}$. This was generated using the tree-level Symanzik-improved gluonic action and non-perturbatively improved Wilson fermions with stout links in the derivative terms (SLiNC action). We use the same relativistic quark action for the (quenched) charm quark with $\kappa_{\text {charm }}=0.11$. Note that since we use the flavor $\mathrm{SU}(3)$ symmetric configurations, the disconnected contributions in the $D_{s} \rightarrow \eta$ 3-point function cancel, when we identify $\eta=\eta_{8}$. The Chroma software package [4] is used for some of the analysis.
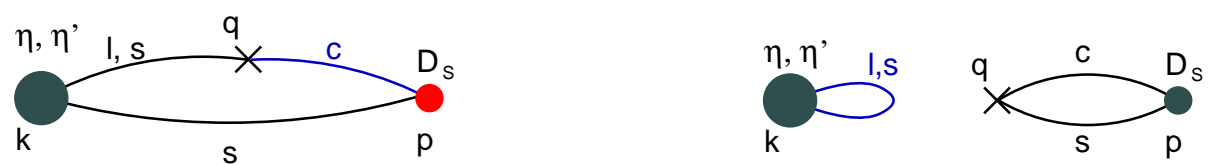

Figure 1: Connected (left) and disconnected (right) diagrams which contribute to $C_{3}\left(t_{f}, t ; \vec{p}, \vec{q}\right)$. We use a stochastic method to estimate the all-to-all propagators, denoted by blue lines. 


\section{Noise Reduction techniques}

In order to calculate the disconnected loop, all-to-all propagators are required. These are estimated using stochastic methods, which involve performing $N$ inversions of the light quark Dirac operator for each configuration; $N$ should be large enough to give sufficiently small stochastic errors relative to the gauge noise. For some quantities the stochastic noise dominates the overall uncertainty and it is important to use efficient noise reduction techniques.

We measure the disconnected "loop"

$$
C_{1}(t ; \vec{p})=\sum_{\vec{x}, \vec{x}^{\prime}, \vec{x}^{\prime \prime}} e^{i \vec{p} \cdot \vec{x}} \operatorname{tr}\left[\gamma_{5} \phi\left(\vec{x}, \vec{x}^{\prime}\right) M^{-1}\left(\vec{x}^{\prime}, t ; \vec{x}^{\prime \prime}, t\right) \phi\left(\vec{x}^{\prime \prime}, \vec{x}\right)\right],
$$

where $M$ is the Dirac operator for a light quark and $\phi$ is a smearing function. The stochastic estimation of the all-to-all propagator $M^{-1}\left(\vec{x}^{\prime}, t ; \vec{x}^{\prime \prime}, t\right)$ involves the following approximation:

$$
M^{-1}=\frac{1}{N} \sum_{i=1}^{N}\left|s_{i}\right\rangle\left\langle\eta_{i}\right|+\mathscr{O}\left(\frac{1}{\sqrt{N}}\right)
$$

where $\left|\eta_{i}\right\rangle$ is a random noise vector and $\left|s_{i}\right\rangle=M^{-1}\left|\eta_{i}\right\rangle$. We use $\frac{1}{\sqrt{2}}\left(\mathbb{Z}_{2}+i \mathbb{Z}_{2}\right)$ complex random numbers for the noise vector. For each $i$ we need to smear both $\left|\eta_{i}\right\rangle$ and $\left|s_{i}\right\rangle\left(\left|\eta_{i}\right\rangle\right.$ must be smeared after solving for $\left|s_{i}\right\rangle$ ) so we need $2 N$ applications of the smearing operator. This significantly increases the computer time needed to calculate the disconnected loop. Time dilution (partitioning) [5] is implemented: the noise vector is only non-zero on one or two time slices.

We test the following three noise reduction techniques.

Spin dilution/partitioning This uses projected noise vectors on a single spinor component and sums over the projections afterwards [5]:

$$
\frac{1}{N} \sum_{a=1}^{4} \sum_{i=1}^{N}\left|s_{i}^{(a)}\right\rangle\left\langle\eta_{i}^{(a)}\right|,
$$

where $\left|\eta_{i}^{(a)}\right\rangle=P^{(a)}\left|\eta_{i}\right\rangle$ is the projected noise vector. It requires $4 N$ inversions but for some quantities the stochastic error is reduced by a factor greater than 2. In addition, we can reduce the cost of smearing because the spin projection $P^{(a)}$ commutes with the smearing of our choice. A naive scaling gives $8 N$ smearing operations, but we only need $5 N$ applications: $4 N$ for $\left|s_{i}^{(a)}\right\rangle$ and $N$ for $\left|\eta_{i}\right\rangle$.

Hopping Parameter Acceleration (HPA) [6] This is based on the following identity

$$
(\kappa D)^{n} M^{-1}=M^{-1}-\kappa D-(\kappa D)^{2}-\cdots-(\kappa D)^{n-1},
$$

where $\kappa D$ is the hopping part of the Dirac operator. Note that the derivative operator satisfies $\operatorname{tr}\left[\gamma_{5} \kappa D\right]=0$ due to the spinor structure so that this term only contributes to the noise. This means that $(\kappa D)^{2} M^{-1}$ represents an improved estimate of $M^{-1}$ (we call it $n=2 \mathrm{HPA}$ ). As long as the smearing is diagonal in spinor space, this is also true for the smeared all-to-all propagator. 
Truncated Solver Method (TSM) For some quantities the ultra violet modes dominate. In these cases, using a small number of CG iterations in the solver for the solution vector $\left|s_{i}\right\rangle$ provides a good approximation, for example, to the disconnected loop [7, 8]. To arrive at an unbiased estimate, a correction term needs to be added to the truncated part:

$$
M^{-1}=\frac{1}{N_{1}} \sum_{i=1}^{N_{1}}\left|s_{\text {trunc }, i}\right\rangle\left\langle\eta_{i}\left|+\frac{1}{N_{2}} \sum_{j=N_{1}+1}^{N_{1}+N_{2}}\right| s_{\text {bias }, j}\right\rangle\left\langle\eta_{j}\right| .
$$

The first term uses the truncated solution $\left|s_{\text {trunc, } i}\right\rangle$, which is cheap to calculate and typically causes the main part of the stochastic error. The second term contains $\left|s_{\text {bias }, j}\right\rangle=\left|s_{\text {conv }, j}\right\rangle-$ $\left|s_{\text {trunc, }, j}\right\rangle$, where $\left|s_{\text {conv }, j}\right\rangle$ is a converged solution. $\left|s_{\text {conv }, j}\right\rangle$ is expensive, and only accounts for a small part of the stochastic error if $\left|s_{\text {bias }, j}\right\rangle$ does not contribute significantly to the observable. Therefore, by tuning parameters $-n$ : number of CG-iterations for the truncated part, $N_{1}$ : number of stochastic noises for the truncated part, $N_{2}$ : number of stochastic noises for the bias part - we can reduce the total calculation cost. We use a CG solver for the truncated solutions and a BiCGstab solver for the converged solutions.

\section{Comparisons}

We investigate the noise reduction techniques using one configuration. We use Wuppertal smearing [9] for the quarks, with parameters which are tuned to minimize the contributions from the excited states to the effective mass.

In Figs. 2 and 3 we plot the stochastic errors for various combinations of the noise reduction techniques. In each case, the computational cost is fixed. The horizontal axes correspond to $n$, the number of iterations of the solver in the TSM. The data at $n=-100$ indicate the results without the TSM. In particular, the red plus symbols ("+") show the results without any noise reduction techniques. For a fixed $n$, we have optimized $N_{1}$ and $N_{2}$ to give the smallest stochastic error under the cost condition

$$
N_{1}\left(n \tau_{\mathrm{CG}}+\tau_{\text {smear }}\right)+N_{2}\left(n \tau_{\mathrm{CG}}+n_{\text {conv }} \tau_{\text {BiCGstab }}+\tau_{\text {smear }}\right)=\text { constant }
$$

assuming the square of error, $\sigma_{\text {stoch. }}^{2}$, to scale according to

$$
\sigma_{\text {stoch. }}^{2}=\frac{f_{1}}{N_{1}}+\frac{f_{2}}{N_{2}}
$$

where $f_{1}$ and $f_{2}$ are the variances of the first and second terms in eq. (2.4), respectively. $n_{\text {conv }}$ is the number of iterations needed to obtain the converged solution. $\tau_{\mathrm{CG}}, \tau_{\mathrm{BiCGStab}}$ and $\tau_{\text {smear }}$ represent the computer time needed for $1 \mathrm{CG}$ iteration, 1 BiCGstab iteration, and smearing, respectively. The optimal ratios of $N_{1} / N_{2}$ are around 1 (10), with (without) smearing.

Although small differences between the results are not significant due to the uncertainty on the stochastic errors, in all cases spin dilution together with HPA (purple squares), gives the minimum error when combined with TSM. Therefore we use this combination in the following analysis.

The gain factor,

$$
g=\frac{\sigma^{2}(\text { without noise reduction })}{\sigma^{2}(\text { with noise reduction })}
$$



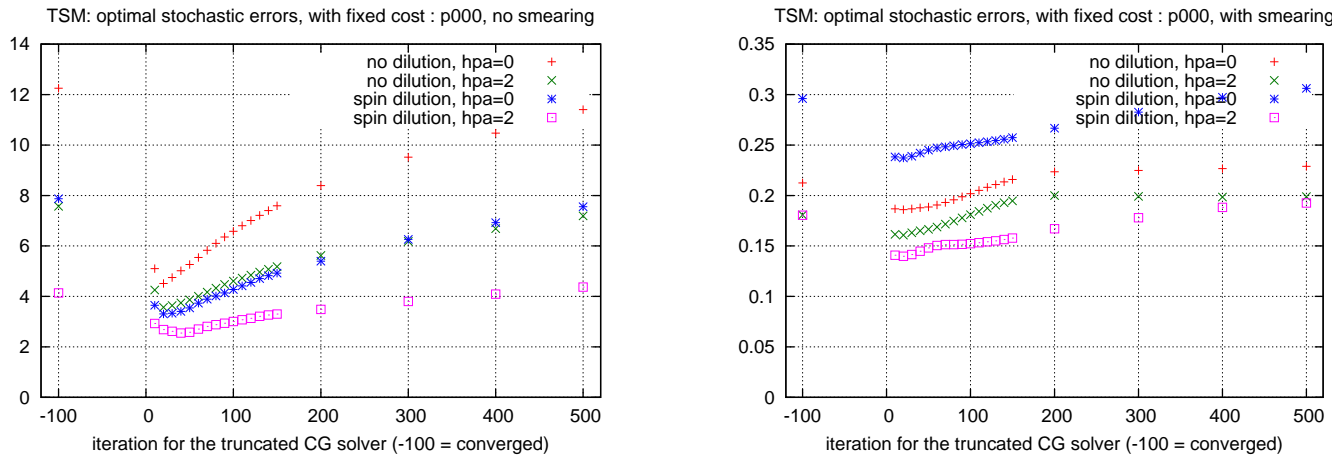

Figure 2: Estimated stochastic errors at fixed cost for $\vec{p}=(0,0,0)$. The horizontal axes are $n$ for the TSM. Data at $n=-100$ are without TSM. Left panel: without smearing. Right panel: with smearing.
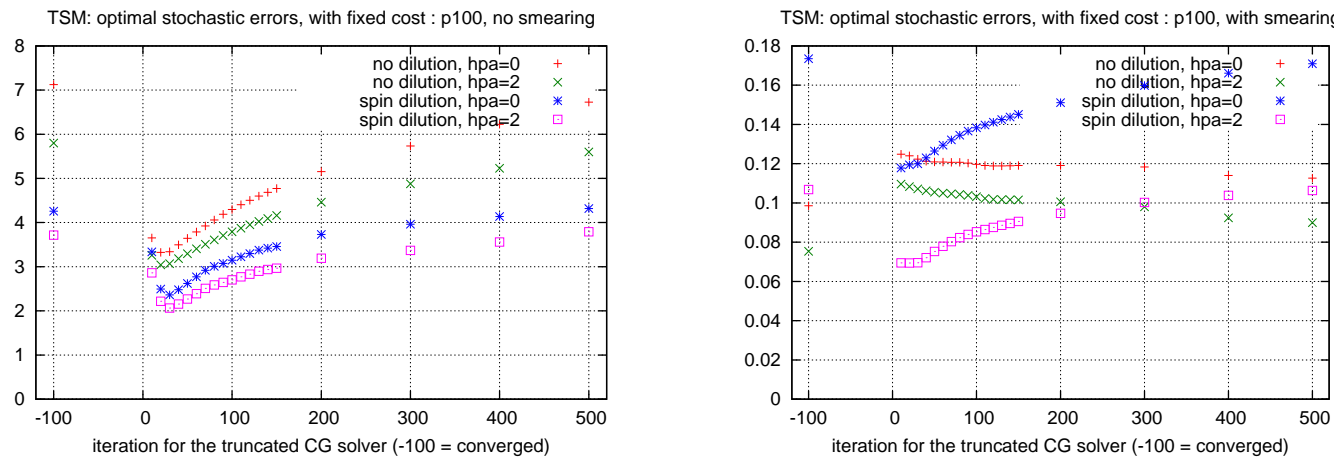

Figure 3: The same as Fig. 2 but for $\vec{p}=(1,0,0)$.

strongly depends on the smearing. Without smearing (left panels), we obtain maximum gain factors of $16-25$, which translates into a reduction of the computational cost of the same magnitude. With smearing, it is only about a factor 2 . This is because the contribution to the error from the bias part (i.e., $\left.f_{2}\right)$ is larger than or of the same magnitude as the truncated part $\left(f_{1}\right)$.

\section{Results}

Having optimized the noise reduction, we can now measure the disconnected contribution to the form factor. For the TSM, we truncate after $n=20 \mathrm{CG}$ iterations and the numbers of noise vectors are $N_{1}=10$ and $N_{2}=20$. A total of 939 configurations were used in the analysis.

Following our previous study [1], we use stochastic techniques for the connected contribution as well. The noise vectors are placed at the sink of the $D_{s}$ meson (denoted by a red circle in Fig. 1). For each configuration, $24 \times 4$ spin diluted noise vectors were computed for the charm quark. In terms of momenta, 57 different combinations of $\vec{p}$ for the $D_{s}$ meson were calculated. Note that a similar calculation with the sequential method would require $57 \times 12$ inversions.

In order to extract the matrix elements in eq. (1.2), we fixed the time separation between the $\eta$ source and the $D_{s}$ sink separately for the connected $\left(t_{f}=24, t_{i}=0\right)$ and the disconnected $\left(t_{f}=24\right.$, $\left.t_{i}=16\right)$ matrix elements. We combine the two contributions afterwards. For the connected part, taking the maximum separation $t_{f}-t_{i}=T / 2=24$ enables us to average over the forward and backward propagations. For the disconnected part, in order to average the forward and backward 

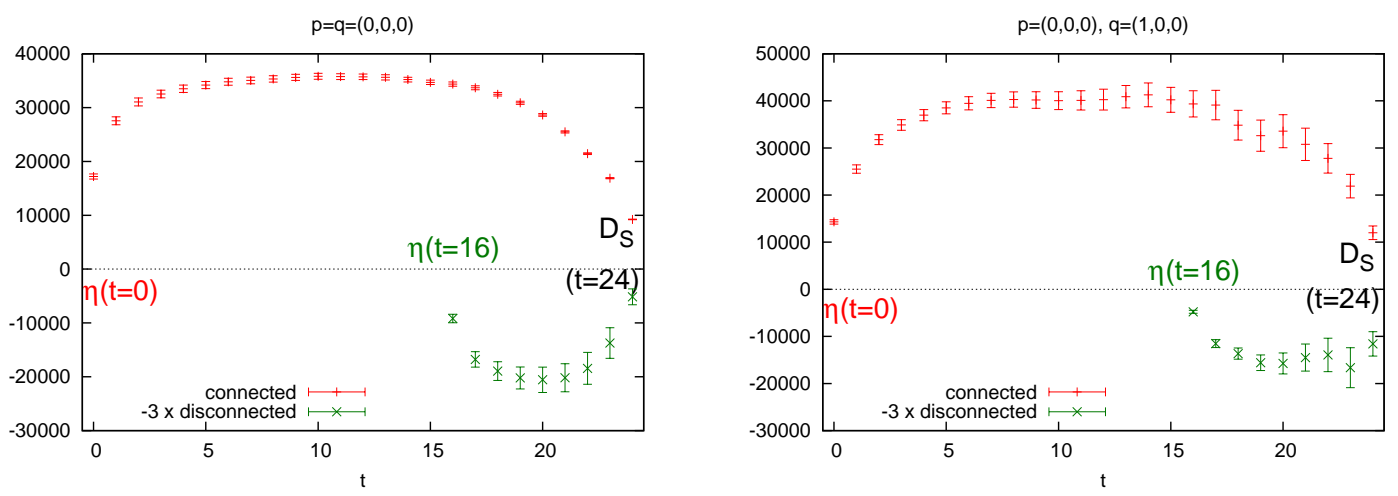

Figure 4: Ratios of 3-point over 2-point functions, $R$ in eq. 1.2, for connected and disconnected parts. $\vec{k}=\vec{q}=(0,0,0)$ for the left panel and $-\vec{k}=\vec{q}=(1,0,0)$ for the right panel.

propagations, the noise vector has a non-zero value at two time-slices separated by 16 time-slices $\left(t_{f} \pm 8\right)$. The usage of different $t_{f}-t_{i}$ for the connected and disconnected 3-point functions is allowed because we have assumed $m_{\eta}=m_{\eta^{\prime}}$ (remember that $m_{u}=m_{d}=m_{s}$ ).

Fig. 4 shows the ratio of the correlation functions, which corresponds to $f_{0}\left(q^{2}\right) / Z_{\eta} Z_{D_{s}}$. The disconnected part is multiplied by 3 because of the 3 light flavors. The errors for the disconnected contribution are small enough to obtain signals, significantly different from zero.

In Fig. 5 we show the form factors for the octet $\left(\eta_{8}\right)$ and singlet $\left(\eta_{1}\right) \eta \mathrm{s}$ :

$$
\begin{aligned}
& \left|\eta_{8}\right\rangle=\frac{1}{\sqrt{6}}(|\bar{u} u\rangle+|\bar{d} d\rangle-2|\bar{s} s\rangle) \quad \text { connected only, } \\
& \left|\eta_{1}\right\rangle=\frac{1}{\sqrt{3}}(|\bar{u} u\rangle+|\bar{d} d\rangle+|\bar{s} s\rangle) \quad \quad \text { connected }-3 \times \text { disconnected. }
\end{aligned}
$$

Preliminary fits to $f_{0}\left(q^{2}\right)$ of the form $f_{0}\left(q^{2}\right)=\frac{f_{0}(0)}{1-b q^{2}}$ give $f_{0}(0)=0.75(3)$ and $f_{0}(0)=0.52(5)$, for $D_{s} \rightarrow \eta_{8}$ and $D_{s} \rightarrow \eta_{1}$, respectively. Also included in Fig. 5 is a value from light cone QCD sum rules for the decay into $\eta$ [10], $f_{0}(0)=0.45(14)$. Due to $\mathrm{SU}(3)$ flavor symmetry the $D_{s} \rightarrow \eta_{8}$ form factor also represents the form factor of $D \rightarrow l v \pi$ and $D \rightarrow l v K$. Note that $f_{0}(0)$ for $\eta_{1}$ is smaller than that for $\eta_{8}$. This is consistent with the form factors for $B \rightarrow \eta, \eta^{\prime}$ [11], which is the heavy quark limit, can be compared to our calculation.

\section{Conclusions}

We tested three methods (and their combinations) of noise reduction techniques for measuring the disconnected contributions to the $D_{s}$ meson semi-leptonic decay form factor. The combination of spin dilution, hopping parameter acceleration and truncated solver method was found to give the biggest gain in computer time. These noise reduction techniques allowed us to measure non-zero contributions to the form factor, on SU(3) flavor symmetric QCDSF $n_{f}=2+1$ configurations. Further studies with non-SU(3) symmetric $n_{f}=2+1$ configurations are planned.

This work was supported by the EU ITN STRONGnet (grant number 238353) and the DFG SFB/Transregio 55. SC acknowledges support from the Claussen-Simon-Foundation (Stifterverband für die Deutsche Wissenschaft). JZ is supported by the Australian Research Council under 


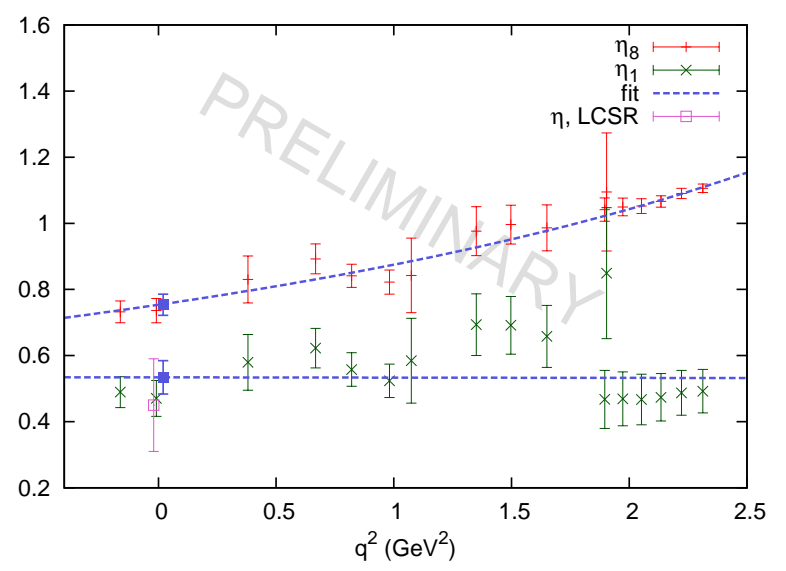

Figure 5: Form factor $f_{0}\left(q^{2}\right)$ for $D_{s} \rightarrow l v \eta_{8}$ and $D_{s} \rightarrow l v \eta_{1}$. Errors are statistical only. A value from QCD light cone sum rules (LCSR) [10] is also plotted. To enhance visibility, the fitted values at $q^{2}=0$ and the LCSR results are slightly shifted to the right and left, respectively.

grant FT100100005. The calculations were performed on the Athene HPC cluster at the University of Regensburg.

\section{References}

[1] R. Evans, G. Bali and S. Collins, Improved semileptonic form factor calculations in lattice QCD, Phys. Rev. D 82 (2010) 094501 [arXiv:1008.3293 [hep-lat]].

[2] H. Na, C. T. H. Davies, E. Follana, P. Lepage and J. Shigemitsu, $D$ semi-leptonic decay form factors with HISQ charm and light quarks, PoS LAT2009 (2009) 247 [arXiv:0910.3919 [hep-lat]].

[3] W. Bietenholz et.al., Flavour blindness and patterns of flavour symmetry breaking in lattice simulations of up, down and strange quarks, Phys. Rev. D84 (2011) 054509 [arXiv:1102.5300 [hep-lat]].

[4] R. G. Edwards and B. Jóo [SciDAC, LHPC and UKQCD Collaborations], The Chroma software system for lattice QCD, Nucl. Phys. Proc. Suppl. 140 (2005) 832 [arXiv:hep-lat/0409003].

[5] S. Bernardson, P. McCarty and C. Thron, Monte Carlo methods for estimating linear combinations of inverse matrix entries in lattice QCD, Comput. Phys. Commun. 78 (1993) 256.

[6] C. Thron, S. J. Dong, K. F. Liu and H. P. Ying, Pade-Z(2) estimator of determinants, Phys. Rev. D 57 (1998) 1642 [arXiv:hep-lat/9707001].

[7] S. Collins, G. Bali and A. Schäfer, Disconnected contributions to hadronic structure: a new method for stochastic noise reduction, PoS LAT2007 (2007) 141 [arXiv:0709.3217 [hep-lat]].

[8] G. S. Bali, S. Collins and A. Schäfer, Effective noise reduction techniques for disconnected loops in Lattice QCD, Comput. Phys. Commun. 181 (2010) 1570 [arXiv:0910.3970 [hep-lat]].

[9] S. Güsken, U. Löw, K. H. Mütter, R. Sommer, A. Patel and K. Schilling, Nonsinglet axial vector couplings of the baryon octet in lattice QCD, Phys. Lett. B227 (1989) 266.

[10] K. Azizi, R. Khosravi and F. Falahati, Exclusive $D_{s} \rightarrow\left(\eta, \eta^{\prime}\right) l v$ decays in light cone $Q C D$, J. Phys. G 38 (2011) 095001 [arXiv:1011.6046 [hep-ph]].

[11] P. Ball, G. W. Jones, $B \rightarrow \eta^{(\prime)}$ form factors in $Q C D$, JHEP 0708 (2007) 025 [arXiv:0706.3628 [hep-ph]]. 